\title{
Entre archivos analógicos y archivos virtuales. Videoarte del sur en la encrucijada
}

\author{
Crescentino, M. Alejandra ${ }^{1}$ \\ ${ }^{1}$ Doctoranda del Programa EALyC, PDIF del Departamento de Lingüística, Lenguas Modernas, \\ Lógica y Fa de la Ciencia, Ta de la Literatura y Literatura Comparada de la Facultad de Filosofía y \\ Letras, Universidad Autónoma de Madrid. FPU-MECD 2016. \\ (UAM, alejandra.crescentino@uam.es)
}

\section{Abstract}

The purpose of this presentation is to reflect on some of the problems affecting analog and virtual archives dedicated to video art in the Southern Cone, in relation to the accessibility, preservation and dissemination of these audiovisual collections in the present.

To this end we refer in the first place to institutional events that promoted the production, circulation and dissemination of audiovisual arts in the last two decades of the 20th century and, simultaneously, fostered the creation of physical archives or video libraries in the region. We refer to the Encuentros Latinoamericanos de Video, Festivales Franco-Chilenos and Franco Latinoamericanos de Video, VideoBrasil and Buenos Aires Video. Secondly, we identify some institutional and academic projects that, since the middle of the year 2000, have given rise to several actions for the valorization and remediation of analogical collections dedicated to video art. Actions through which it has been possible to give visibility, accessibility and survival to some collections. Finally, under the premise of problematizing the initiatives that safeguard this audiovisual culture and its difficulties to survive, common problems to audiovisual archives in the present are pointed out, and the answers articulated by some research projects to face them are presented.

Keywords: Audiovisual archives, video art, remediation projects, Southern Cone.

\section{Resumen}

Esta presentación tiene como objetivo reflexionar sobre algunas problemáticas que afectan a los archivos analógicos y virtuales dedicados al videoarte en el Cono Sur, en relación con las prestaciones de accesibilidad, conservación y difusión de estos acervos audiovisuales en el presente. 
Para ello se alude, en primer lugar, a eventos institucionales que fomentaron la producción, circulación y difusión de las artes audiovisuales en las dos últimas décadas del siglo $X X ; y$, simultáneamente, propiciaron la creación de archivos físicos o videotecas en la región. Nos referimos a los Encuentros Latinoamericanos de Video, los Festivales Franco-Chilenos y Franco Latinoamericanos de Video, VideoBrasil y Buenos Aires Video. En segundo lugar, se identifican algunos proyectos institucionales $y$ académicos que desde mediados del año dos mil, han dado lugar a diversas labores de puesta en valor y remediación de acervos analógicos dedicados al videoarte. Acciones mediante las cuales se ha logrado dar visibilidad, accesibilidad y supervivencia a estos patrimonios. Finalmente, bajo la premisa de problematizar las iniciativas que dan resguardo a esta cultura audiovisual y sus dificultades de permanencia, se puntualizan problemas comunes a los archivos audiovisuales en el presente y se presentan las respuestas articuladas por algunos proyectos de investigación para hacerles frente.

Palabras clave: Archivos audiovisuales, videoarte, proyectos de remediación, Cono Sur. 


\section{Introducción}

Esta presentación tiene por objetivo reflexionar sobre algunas problemáticas que afectan a los archivos analógicos y virtuales dedicados al videoarte en el Cono Sur, en relación con las prestaciones de accesibilidad, conservación y difusión de estos acervos audiovisuales en el presente.

Para dar lugar a esta discusión se alude, en primer lugar, a eventos institucionales que fomentaron la producción, la circulación y la difusión de las artes audiovisuales en las dos últimas décadas del siglo XX; $\mathrm{y}$, simultáneamente, propiciaron la creación de archivos físicos, o videotecas, en la región. En segundo lugar, se identifican algunos proyectos institucionales y académicos que, desde mediados del año dos mil, han dado lugar a diversas labores de puesta en valor y de remediación de acervos analógicos dedicados al videoarte. Acciones mediante las cuales se ha logrado dar visibilidad, accesibilidad y supervivencia a estos patrimonios. Finalmente, bajo la premisa de problematizar las iniciativas que dan resguardo a esta cultura audiovisual y sus dificultades de permanencia, se puntualizan problemas comunes a los acervos audiovisuales en el presente y se presentan las respuestas articuladas por algunos proyectos de investigación para hacerles frente.

En cuanto a los aspectos metodológicos, esta aproximación se basa en fuentes clásicas (bibliográficas y documentales) y electrónicas (sitios web, bases de datos y archivos en línea, plataformas de proyectos de investigación, etc.). Asimismo, se han consultado presencialmente centros documentales y archivos en Chile, Argentina y España. Frente a la imposibilidad de abarcar todas las instituciones y archivos dedicados al videoarte en el Cono Sur, fue necesario establecer un recorte que permitiera un acercamiento más concreto al tema. Para ello, se hizo foco en algunos archivos vinculados a cinco eventos pioneros del videoarte en la región: los Encuentros Latinoamericanos de Video, los Festivales Franco-Chilenos y Franco Latinoamericanos de Video, el festival VideoBrasil y, en último lugar, Buenos Aires Video. A partir de ellos se explora su relación con proyectos académico-institucionales que proponen posibles maneras de remediar y dar visibilidad a los archivos nacidos analógicos.

En relación con el marco de referencia general, las contribuciones teóricas del campo del New Media ofrecen herramientas conceptuales que resultan apropiadas para nuestro abordaje. Por un lado, adscribimos a la noción de remediation (Bolter y Grusin, 2000), que alude al poder de los medios digitales para remediar medios analógicos a través de un proceso de remodelado (refashioning) o transcodificación (Manovich, 2001,p.47). Este argumento nos permite concebir los archivos y repositorios digitales institucionales del presente, como extensiones remediadas de archivos y videotecas analógicas. Tras el advenimiento del mundo digital han surgido nuevas plataformas hipermedia - sin base en espacios físicos preexistentes - que operan igualmente bajo 
esta lógica: remediando obras audiovisuales analógicas y ofreciendo contenidos hipermediados.

Por otra parte, esta aproximación considera aportes críticos y teóricos dedicados a explorar los cruces entre las instituciones y las nuevas tecnologías, y el rol de estas tecnologías en la difusión del patrimonio cultural. Perspectivas que atienden al impacto de los procesos de digitalización y globalización en el campo de la creación cultural contemporánea, transformando las prácticas artísticas y el aparato material e institucional en donde se insertan (Sarlo, 1994; Steyerl, 2014).

En relación con lo antedicho, el investigador argentino Jorge La Ferla identificó diversas dificultades vinculadas a la ausencia de acervos comprensivos para trazar una memoria de las artes audiovisuales en América Latina. En sus reflexiones en torno a "una praxis de archivos del sur", señalaba cuestiones muy pertinentes al respecto de la circulación y la conservación de las obras audiovisuales. Entre ellas, la escasez de proyectos de carácter público y privado encaminados a la conformación de archivos institucionales panorámicos de la región ${ }^{1}$. También aludía al rol fundamental que tuvieron algunas muestras itinerantes de vídeo y cine experimental en la conformación de acervos, la reconstrucción teórica y la recuperación matérica de piezas que se creían perdidas. Subrayaba que, ante la ausencia de políticas públicas de protección patrimonial consistentes, en el Cono Sur, algunas instituciones y agentes habían asumido la tarea de conservar y actualizar las obras tecnológicas, que de otra manera quedarían inaccesibles. Finalmente destacaba otro orden de dificultades en relación con los acervos existentes. Entre ellos, el estatus patrimonial indefinido de las piezas incorporadas a las instituciones, la fragmentariedad de esos patrimonios y como consecuencia de ello, la dificultad de realizar lecturas interpretativas de conjunto (La Ferla, 2010 a y b). Estas observaciones, realizadas hace más de diez años, constituyen un punto de partida para ofrecer una relectura de estas problemáticas en torno a los archivos analógicos y digitales del presente.

\section{Festivales y archivos de videoarte en el Cono Sur: legados del pasado analógico}

Resulta necesario realizar una sucinta mirada retrospectiva para comprender el estado actual de los archivos institucionales que poseen obras videográficas en soportes analógicos. Para ello destacaremos algunos festivales dedicados a esta disciplina, de trascendencia regional e internacional, realizados desde los años ochenta en el Cono

\footnotetext{
${ }^{1}$ Con la salvedad del Acervo de la Associação Cultural Videobrasil, al cual La Ferla ha aludido reiteradamente como un caso excepcional.
} 
Sur. De forma paralela a los eventos, varias de las instituciones organizadoras generaron acervos dedicados al videoarte. Videotecas, mediatecas y archivos conservaron diversos materiales audiovisuales, en donde se yuxtaponían copias de las obras participantes en los festivales, con registros de actividades llevadas a cabo por la institución y obras videográficas de referentes internacionales.

Por supuesto, el uso del vídeo como soporte y medio de creación artística es anterior a la década del ochenta. Sus antecedentes se remontan a principios de los años setenta y están ligados a unas pocas instituciones. Entre ellas, el Centro de Artes y Comunicación de Buenos Aires (CAYC) y el Museu de Arte Contemporânea de la Universidad de São Paulo (MAC-USP), con trayectorias paradigmáticas a este respecto. Ambas entidades pusieron a disposición de los artistas equipos porta pack, y de este modo fomentaron la experimentación a través de las nuevas tecnologías. Bajo dirección de Jorge Glusberg, el CAYC se convirtió en una de las primeras instituciones de la región en organizar exposiciones internacionales de vídeo e impulsar la producción en tal soporte. Lamentablemente ninguna de las obras expuestas en estas exposiciones, ni las producidas desde el CAYC, ha sobrevivido hasta nuestros días. Esto se debió -como subraya Alonso-, a que el costo elevado de estas novedosas tecnologías dificultó la posesión de copias de resguardo (2015, p.99). Bajo la gestión de Walter Zanini, el MAC USP también ocupó un rol pionero al crear un Sector de Vídeo y una sala dedicada exclusivamente a la programación de vídeo -el Espaço B- en 1977. El carácter universitario de este espacio dio lugar, recientemente, a la articulación de un proyecto de investigación dedicado a este período que ha sido plasmado parcialmente en la exposición "Vídeo_MAC". Tal inciativa, de gran valor patrimonial y documental, permite conocer producciones brasileñas en videoarte, totalmente desconocidas hasta el momento ${ }^{2}$.

Más allá de estos ejemplos puntuales, el vídeo no logró un alcance y presencia significativos hasta la década del ochenta. Momento que coincidió con un proceso de gradual ampliación de la base social de los usuarios del soporte electromagnético, estrechamente ligado a la expansión de los medios de comunicación masiva y las nuevas tecnologías de la mano de capitales transnacionales. Como es lógico, tales circunstancias tuvieron impacto en el mundo del arte, y para mediados del decenio el video había entrado - aunque tímidamente- en diversos espacios institucionales a

\footnotetext{
2 Se quiere destacar la tarea que subyace a "Vídeo_MAC", articulada como propuesta expositiva en línea en 2020. El recorte se enfoca en las producciones videográficas realizadas durante los años 1977 y 1978 para el Espaço B del MAC-USP, de la mano de una invaluable remediación de dieciocho obras de vídeo arte, ahora enteramente disponibles a través del sitio web del proyecto. Roberto Moreira S. Cruz se ubica al frente de esta propuesta de comisariado, que es resultado de su investigación posdoctoral para la Universidade de São Paulo (Museu de Arte Contemporânea da Universidade de São Paulo, s.f.).
} 
través de ciclos, jornadas y encuentros, fomentados en gran parte por artistas, productores y realizadores independientes. Como caso ilustrativo de tal convergencia, situamos a los Encuentros Latinoamericanos de Video (1988-1993), impulsados por productores independientes de toda América Latina. Entre el centenar de asociaciones y colectivos al frente de estos encuentros, se encontraba el Centro de Medios Audiovisuales (CEMA), una productora uruguaya que destacó por su amplia producción audiovisual, y fue objeto de un proyecto de investigación académica ulterior que será referido en este trabajo.

También en los espacios institucionales el video cobró fuerza como disciplina artística y autónoma, apuntalada en un circuito internacional de eventos dedicados al videoarte. Se crearon festivales, muestras y premios, organizados por instituciones de referencia en el ámbito local y organismos de financiación extranjera - particularmente a través de la cooperación francesa y la española-. Estas articulaciones fortalecieron los vínculos regionales preexistentes, y estimularon la producción y la reflexión sobre el videoarte del Cono Sur.

Retomando lo señalado por La Ferla, la proliferación de eventos en torno al video en este período fue un factor de peso para la conformación de archivos audiovisuales. Asimismo, las videotecas se convirtieron en espacios muy apreciados, donde era posible consultar materiales audiovisuales en diversos formatos magnéticos u ópticos.

Constituyen ejemplo de estas iniciativas los Festivales Franco-Chilenos (1981-1994) y Franco-Latinoamericanos de Videoarte (1992-1996), que se afianzaron gracias al sostén brindado por el Ministerio de Asuntos Exteriores de Francia y la participación de numerosas instituciones locales. Por más de una década, Santiago de Chile fue la capital de un evento que atrajo la atención de sus vecinos del sur. A principios de los años noventa, el festival amplió su alcance regional al convertirse en un evento multisede que se extendió por Argentina, Montevideo, Brasil y Colombia. Como resultado proyectado al presente, cada institución participante conformó un acervo parcial del mismo, resguardando piezas videográficas, vestigios y/o fuentes documentales. Según las políticas de conservación aplicadas por cada institución, los mismos se encuentran accesibles para consulta presencial o virtual ${ }^{3}$.

En Brasil, el precursor en este ámbito fue el festival VideoBrasil (1983-Presente). Sus primeras ediciones se realizaron en el Museu da Imagem e do Som (MIS), y más tarde fueron organizadas por la Associação Cultural Videobrasil junto al Servicio Social del

\footnotetext{
${ }^{3}$ Entre estos espacios de consulta pública figuran la Biblioteca y Centro de Documentación del Museo Nacional de Bellas Artes de Chile; el Centro de Documentación de las Artes Visuales de Chile (CEDOC); el Centro de Documentación y Archivo Centro Cultural Rector Ricardo Rojas de la Universidad de Buenos Aires (CCRR-UBA), la Biblioteca Nacional de Colombia y el Centro de Memoria e Información del Museu da Imagem e do Som (MIS) de São Paulo.
} 
Comercio $(\mathrm{SeSC})^{4}$. A partir de la década del noventa, el festival declaró la expansión de su alcance de acción a todo el sur geopolítico y fundó la asociación para albergar un patrimonio cada vez más amplio de fondos audiovisuales, documentales y publicaciones (Associação Cultural Videobrasil, s.f). Gracias a esta política institucional, la tarea desarrollada por esta entidad ha sido de gran valor, y no tiene parangón con otras asociaciones similares dentro de Brasil o en el Cono Sur.

También a fines de los ochenta, apareció a la zaga el festival Buenos Aires Video (19892013) en el seno del Centro Cultural del Instituto de Cooperación Iberoamericana (ICI) en Buenos Aires ${ }^{5}$. Las primeras ediciones, comisariadas por Carlos Trilnick, se realizaron bajo el formato "muestra" y proponían un panorama videográfico local. A partir de la sexta edición asumieron un carácter competitivo e incluyeron selecciones de otros países, lo que estimuló la circulación de las producciones argentinas por la región. Con

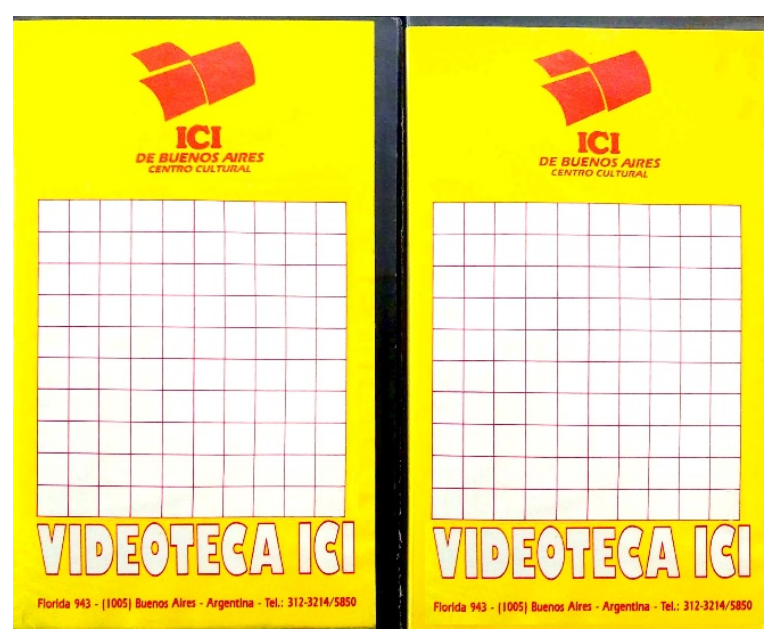
la inauguración del centro se creó también una videoteca con equipos para la consulta in situ (Fig.1). Sus fondos dedicados al videoarte español, argentino y latinoamericano se vieron engrosados paulatinamente con el ingreso de las obras seleccionadas en Buenos Aires Video. Sin embargo, tras el giro digital, tanto el festival como la videoteca dieron por concluida su tarea, y esta última dejo de ser accesible al público (Crescentino, 2021).

Fig. 1: Cajas de videocassettes de la videoteca del ICI. Fondo: Biblioteca del CCEBA. Fotografía: Alejandra Crescentino.

${ }^{4}$ Bajo dirección de la seteropolitana Solange Farkas desde sus comienzos a la actualidad, curadora de estos eventos y comprometida con la conservación de su acervo.

${ }^{5}$ El mismo dependía del Instituto de Cooperación Iberoamericana de Madrid, adscripto a la Agencia Española de Cooperación Internacional para el desarrollo (AECID). EI ICI de Buenos Aires fue el primer centro de la Red de Centros de AECID en Latinoamérica, y se inauguró en 1988 con la exposición "La imagen sublime. Video de Creación en España (1970-1987)", comisariado por Manuel Palacio. 


\section{Cultura digital y el "giro archivístico"}

El advenimiento de la cultura digital, en red y globalizada, modificó las formas de uso y acceso a la información de forma decisiva. Con el "pasaje hacia el mundo de las pantallas" se produjo un vertiginoso salto cultural que cambió el registro de lo cotidiano y mediatizó la vida (Sarlo, 1994).

Se inició, así, un proceso gradual de informatización institucional, en el cual las herramientas informáticas se convirtieron en aliadas para la gestión documental de archivos y colecciones, y se desplazó a los sistemas y tecnologías analógicas. Para el caso de las videotecas y archivos audiovisuales, equipados con tecnologías y soportes analógicos, esta transformación provocó su desactivación como espacios de referencia y sus materiales de consulta se tornaron "objetos arqueológicos". Y, si bien muchas instituciones conservaron estos fondos (compuestos, mayoritariamente, por soportes U-MATIC, VHS, Betamax, Betacam, entre los más populares), muy pocas se ocuparon de llevar adelante un proceso de digitalización de los mismos.

Por otra parte, el giro digital impactó en las formas de mirar y pensar el fenómeno del videoarte regional. Hubo un pasaje desde los relatos de las escenas nacionales delineados por eventos durante los años ochenta y noventa-, a la construcción de relatos desde las escenas locales, conectadas e inmersas en el mapa global del mundo en red. Algunas instituciones comenzaron a volcar gran cantidad de contenidos, y a comunicar actividades, en sus sitios web institucionales. Simultáneamente se generaron proyectos de investigación que se orientaron a explorar los caminos dispersos de la relación arte-tecnología en el cono sur, bajo un formato de trabajo asociativo entre institución y academia.

Como resultado de este interés, desde mediados del año dos mil comenzaron a emerger un número significativo de plataformas dedicadas al videoarte regional. Tales hipermedia, vinculados a proyectos académicos e institucionales -y articulados como archivos o bases de datos en línea-, han formulado nuevas lecturas sobre el videoarte latinoamericano. En ocasiones, también han venido acompañadas de propuestas expositivas y editoriales bajo el objetivo de dar visibilidad a obras en formatos que habían quedado extintos.

\subsection{Remediaciones del pasado analógico}

Aunque las tecnologías digitales determinaron la obsolescencia de los soportes analógicos, el alcance logrado por proyectos de remediación de este pasado analógico es impensable, en términos de organización, sin las herramientas digitales. 
Como señalan Bolter y Grusin, la búsqueda de intermediación atraviesa toda la historia del desarrollo de los medios de comunicación en Occidente ${ }^{6}$. Inmersos en esta lógica, los nuevos medios ampliaron esta capacidad de mediación gracias a su poder de incorporar la casi totalidad de los soportes y tecnologías anteriores. Para aludir a esta agencialidad de los medios, los autores han propuesto la noción de remediación, caracterizada como reforma ("remediation as reform") y como "reparación" de un medio anterior (2000, p.52-62). En este sentido, la remediación puede ser entendida de dos maneras. Por un lado, como una forma de mediación de medios anteriores, una intermediación. Y, por otro, como una reparación, una forma de precaver un peligro, en este caso, la desaparición de obras nacidas bajo formatos que se vuelven obsoletos.

En el caso de los archivos analógicos estas "remediaciones" han sido articuladas a partir de valiosos proyectos de pesquisa que rescatan de la obsolescencia o la invisibilidad acervos audiovisuales vinculados al recorrido de festivales e instituciones antes propuesto. Un aspecto que se quiere enfatizar, es que todos estos desarrollos no solo plantean la remediación digital de contenidos analógicos audiovisuales o documentales, sino que encaran acciones para revitalizar estos acervos a través de publicaciones, exposiciones, debates y ciclos, entre otras propuestas.

Uno de los primeros en aparecer fue el "Proyecto Patrimonio audiovisual chileno UMATIC", encabezado por el documentalista e investigador Germán Liñero.

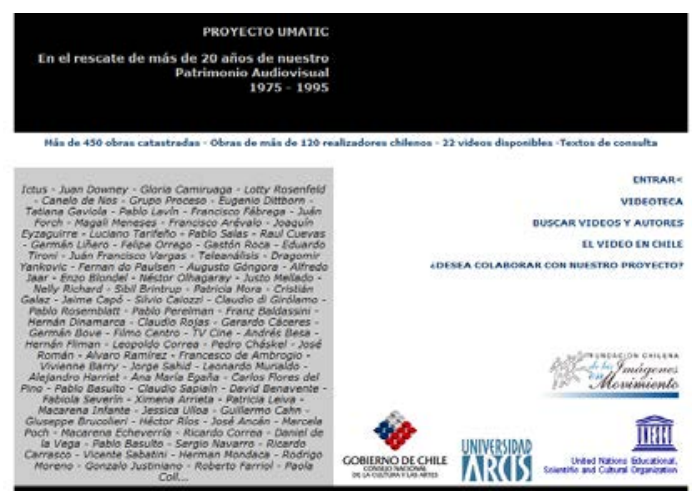

Fig. 2: Captura de pantalla del sitio web del Proyecto U-MATIC de Germán Liñero.

\footnotetext{
${ }^{6}$ Los autores aluden al recorrido trazado en el libro de McLuhan en Understanding Media: The Extensions of Man (1964) en sus argumentaciones (Bolter y Grusin, 2000, p.77).
} 
La iniciativa proponía abordar la exploración y registro de obras realizadas bajo este formato en Chile entre los años 1975 y 2000, de modo que abarcaba las producciones presentadas en los Festivales Franco-Chilenos, Franco-Latinoamericanos y los Encuentros Latinoamericanos de Video, entre otros. Los resultados de esta investigación se articularon en tres ejes: la concreción de la "Muestra U-MATIC", realizada en el Museo de Arte Contemporáneo de Santiago en 2005; la publicación del libro "Apuntes para una historia del vídeo en Chile" en 2010; y la creación de un sitio web (Fig.2). Este último, al presente extinto, exhibía fragmentos digitalizados de más de una veintena de piezas audiovisuales escogidas, y más de cuatrocientas fichas técnicas de títulos con reseñas sobre sus autores ${ }^{7}$.

En el año 2015, los acervos chileno y argentino de los festivales Franco Chilenos y Franco-Latinoamericanos se revitalizaron gracias a la donación efectuada por PascalEmmanuel Gallet -agregado cultural francés e impulsor de los festivales-. Este legado generó revisiones y una reflexión retrospectiva sobre el impacto de los festivales en las escenas locales. En Chile, se integró al Centro de Documentación del Museo Nacional de Bellas Artes, en el marco de las celebraciones de la $12^{\text {va }}$ Bienal de Artes Mediales (Gallet, 2015). En Argentina, se incorporó al Instituto de Investigaciones en Arte y Cultura Dr. Norberto Griffa (IIAC) de la Universidad Tres de Febrero, y dio lugar a la muestra "La imagen que desborda: viaje, diario y videoarte" de 2016 (Zuzulich, 2016). La exposición se convirtió en una oportunidad única para revisitar las obras y los documentos que componen el Fondo Pascal Gallet y el Archivo IIAC, particularmente los intercambios de miradas que proponen los video-diarios de viaje en realizados por artistas franceses, sudamericanos y bálticos involucrados en los festivales mencionados.

En 2008, se orquestó otro importante proyecto que proponía el rescate del archivo UMATIC del CEMA en Uruguay. Como se mencionó antes, el Centro de Medios Audiovisuales fue una productora audiovisual uruguaya que concretó una significativa producción videográfica desde 1982 y tuvo un rol muy activo en la organización de los Encuentros Latinoamericanos de Video. Un equipo coordinado por la investigadora Mariel Balás - con el apoyo de la Universidad de la República y con fondos del Instituto del Cine y el Audiovisual (ICAU) - , encaró la digitalización del archivo de la productora a fin de salvarlo de un deterioro irremediable. Posteriormente se publicó un libro que recorría tanto lo realizado por este colectivo como la trayectoria del proyecto de

${ }^{7}$ El proyecto obtuvo el financiamiento del Consejo Nacional de la Cultura y las Artes de Chile (FONDART); el patrocinio de UNESCO-Chile, el Instituto Chileno Francés de Cultura, y el auspicio de la Universidad ARCIS y la Fundación Chilena de Imágenes en Movimiento. Información obtenida en: http://www.umatic.cl/ [sitio web no disponible en línea]. 
investigación bajo el título "CEMA: Archivo, video y restauración democrática" (Tadeo Fuica y Balás, 2016).

Por su parte, la asociación cultural responsable de la organización del Festival VideoBrasil ha orientado sus esfuerzos a mantener una sede física para resguardar su acervo, compuesto por miles de piezas audiovisuales y documentales, catalogadas. Entre ellas, en torno a mil quinientas son obras videográficas que pasaron por el festival. A partir de 2007, se inició un proceso de digitalización de las obras que componían estos fondos y se consideró la posibilidad de su publicación en línea, tarea que finalmente no se llevó a cabo por las limitaciones establecidas por los derechos autorales (Pato, 2014). No obstante, la opción de consulta del catálogo de su sitio web institucional permite acceder a fichas que ofrecen una breve sinopsis, una imagen y el detalle de su participación en VideoBrasil (Fig.3) (Associação Cultural Videobrasil, s.f). Asimismo, la asociación no ha cesado de impulsar actividades de relectura y reactivación de su colección, que incluyen exposiciones, publicaciones, residencias artísticas y de investigación, entre otras.

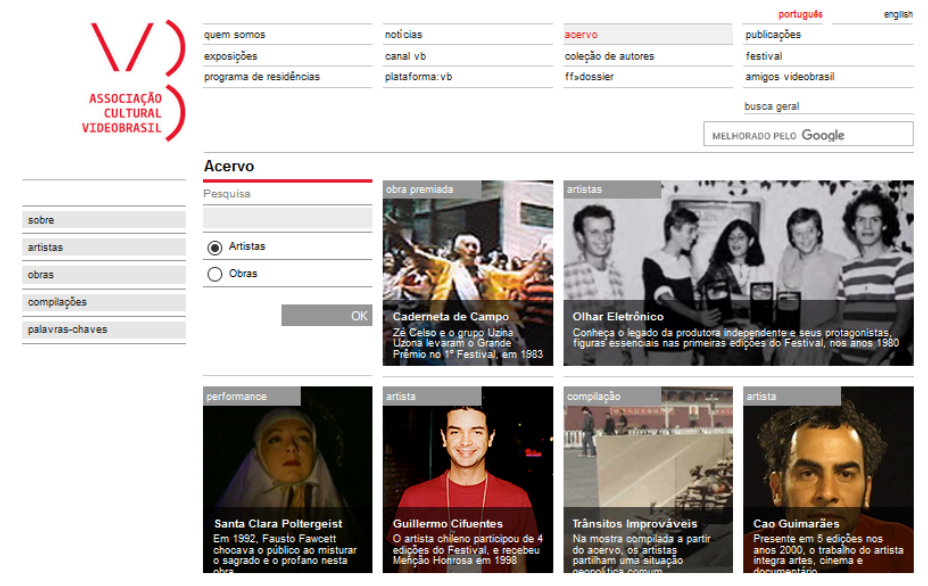

Fig. 3: Captura de pantalla del Acervo en línea de la Asociación Video Brasil.

En estrecha conexión con la necesidad de dar visibilidad a obras que habían transitado por eventos tales como Buenos Aires Video, nació el proyecto ARCA Video Argentino. La iniciativa surgió en 2008 con el objetivo de remediar digitalmente las piezas de una historia del vídeo argentino que abordaba las obras exhibidas en Buenos Aires desde la década del ochenta a la actualidad, y bajo el deseo de extenderse a otras ciudades argentinas. Coordinado por la investigadora Mariela Cantú, contó con el apoyo y la financiación de la Universidad Nacional de La Plata y el Fondo Nacional de las Artes en las distintas fases del proyecto (Cantú, 2011). A principios de 2021, se presentó el 
nuevo diseño de interfaz de su sitio web, en donde se entrelazan hipervínculos a contenidos fuera de la web, con vídeos incrustados provenientes de otras plataformas (Cantú, s.f.). El carácter colaborativo y sin fines de lucro de la plataforma apela al interés de los propios autores para poner en circulación obras que permitan completar la historia del vídeo en Argentina. De este modo, se evitan algunas de las dificultades que plantea el copyright a la hora de hacer accesibles estos contenidos.

A pesar de que existen muchos más, los casos presentados admiten una aproximación a iniciativas que se han propuesto afrontar los desafíos implicados en el rescate de las historias vinculadas a los medios inestables. Asimismo, nos referimos "archivos virtuales" porque no todas las obras y documentos publicados en estas plataformas poseen un respaldo físico, es decir, gran parte de la información presentada sólo existe en formato digital. Por otro lado, los acervos físicos (parcialmente) digitalizados y convertidos en archivos virtuales, facilitan la circulación de obras y fondos documentales sobre el videoarte del Cono Sur. En este sentido, tanto aquellos que cuentan con soporte físico como los que no, se transforman en formas de archivo resistentes (Steyerl, 2014, p.38) que mantienen con vida las obras y hacen posible la formulación de nuevas aproximaciones y narrativas sobre el vídeo de la región.

\section{Videoarte y archivo: una relación problemática}

Tras esta cartografía puntual de festivales de videoarte, archivos audiovisuales y proyectos de remediación, es posible señalar algunas problemáticas comunes a los espacios que resguardaron obras videoartísticas originalmente analógicas. Las cuestiones que se proponen a continuación, asimismo, no son exclusivas a los archivos analógicos y digitales de videoarte del Cono Sur, pero igualmente plantean desafíos metodológicos y dificultades técnicas a los investigadores interesados en abordar el estudio de estos corpus. En este sentido, se puntualizan algunos de estos problemas y las respuestas articuladas por los proyectos de investigación referidos.

\subsection{Dispersión geográfica}

En este caso hacemos referencia tanto a instituciones que poseen obras videográficas en soportes analógicos o digitales, y materiales documentales sobre las mismas que sólo pueden consultarse in situ. La distancia geográfica entre archivos hace que las posibilidades de consulta a los acervos audiovisuales en el Cono Sur sean escasas o intrincadas, y convierten en tarea ímproba el abordaje de las historias locales y regionales. Asimismo, mientras que muchas de las instituciones mencionadas ofrecen registros parciales de catalogación en línea, otras ni siquiera ofrecen esta información. 
Aunque se presenta como mejor respuesta, la remediación digital de obras videográficas en soportes analógicos y su puesta en circulación en diversas plataformas en línea no resuelve esta problemática. La dispersión de contenidos audiovisuales y la dificultad de encontrar un corpus de obras reunidas en un mismo lugar también existe en el mundo en red. No obstante, la mayoría de los proyectos de investigación que cuentan con archivos en línea también facilitan los cruces con otras plataformas audiovisuales y con sitios web de proyectos afines o conectados, aspecto que facilita parcialmente la tarea exploratoria.

\subsection{Lagunas documentales en archivos}

Los criterios de conformación de archivos y videotecas de videoarte no han sido estables, como tampoco lo han sido los criterios de registro y catalogación de las piezas incorporadas. Acompañando las obras audiovisuales, suelen converger registros y diversos documentos institucionales en relación con esas obras. Habitualmente esta información se encuentra sistematizada de alguna manera, aunque muchas veces es parcial. Aunque el problema mayor surge cuando las instituciones carecen de registros, documentación o copias de los materiales editados o producidos por la propia institución. Una hipótesis factible sobre estas lagunas es que en el momento de eclosión del videoarte - durante las décadas del ochenta y noventa-, muchos de estos espacios simplemente no contemplaban la posibilidad de implementar prácticas de documentación y archivo sistemático - de esta u otras manifestaciones artísticas basadas en el tiempo-y, por ende, el resguardo de estos materiales quedó a merced de la implicación y competencias archivísticas de los responsables a cargo.

Quizás la única solución frente a los problemas figurados por la dispersión y las lagunas en archivos sea apoyar la generación de proyectos de investigación situados y locales, en contacto asiduo con los acervos institucionales, capaces de construir lecturas críticas y profundizar en la indagación de estos materiales en diversos archivos. Solamente la puesta en circulación de múltiples proyectos que propongan este tipo de abordajes hará posible la articulación de nuevas investigaciones panorámicas regionales.

\subsection{Status de obra y copyright}

Como política recurrente, los eventos y festivales de videoarte estipularon en las bases de sus convocatorias que una copia del trabajo seleccionado debía quedar a disposición de la institución organizadora. Con su participación, el autor consentía automáticamente que su obra podía ser utilizada con fines culturales-no comerciales, o destinada a videotecas o espacios de consulta. De este modo, el acopio de estos 
materiales fue creciendo paulatinamente, según el éxito de los eventos organizados. Para las instituciones, el vídeo era fácil de exhibir y de guardar, y para los videoartistas, integrarse en sus acervos aseguraba la circulación de sus producciones. Así las piezas presentadas actuaban como "obras" al ser proyectadas en los eventos, pero luego recuperaban su status de "copia" en el archivo. Cuando los festivales dejaron de celebrarse y las videotecas cerraron sus puertas al público, los soportes quedaron en un lugar de indefinición patrimonial, agravado, en ocasiones, por las lagunas en su documentación. En otras palabras, aunque existe algún documento que da constancia de su ingreso a la institución, su condición entre objeto mueble, obra patrimonial y fondo documental lo mantienen en estado de status indeterminado, y, por lo mismo, cualquier tipo de registro audiovisual para su publicación incurriría en problemas de copyright.

En este tipo de dificultades se han visto involucradas todas las iniciativas orientadas a remediar y poner en circulación a través de plataformas en línea estos contenidos. En este caso, las soluciones implementadas por proyectos como ARCA consisten en convertirse en plataformas colaborativas abiertas, que se amplían a partir de las cesiones voluntarias o generando hipervínculos con sitios web y plataformas de los autores de las obras audiovisuales.

\subsection{Inaccesibilidad y pérdida de obras en medios inestables}

Mientras los archivos y videotecas se mantuvieron activos, las obras videoartísticas se mantuvieron vivas, y se las siguió incluyendo en múltiples propuestas. Con la transformación digital, los equipamientos tecnológicos analógicos, dispuestos para acceder a las obras, fueron quedando obsoletos, y las obras se tornaron inaccesibles. Como ya se ha señalado, son muy pocos los archivos y videotecas institucionales que se han ocupado de llevar adelante tareas de digitalización de sus acervos. Esto se ha excusado apelando a cuestiones de copyright y costes elevados de digitalización para las instituciones, y, si bien, algunos autores se han ocupado individualmente de realizar esta tarea, otras obras se han perdido o resultan hoy inaccesibles.

No obstante, existen otros motivos de peso que han marcado este estado de situación, vinculado a las volubles políticas de institucionalización en la región y a los desafíos de conservación que plantean los soportes basados en medios inestables. El videoarte ha desempeñado tradicionalmente un rol de outsider en museos y espacios culturales. $\mathrm{Y}$, salvo cierta demostración eventual de interés - genuina u oportunista - sujeta a determinada gestión, no se han creado políticas públicas de protección específica, ni se han sostenido en el tiempo tareas de puesta en valor de estas producciones. 
Por otro parte, se parte de la premisa de que tanto los soportes analógicos como los digitales son inestables, y si bien su remediación digital permitiría extender su supervivencia, es necesario acompañar estas acciones con otras que las doten de significado y aseguren diversas maneras de persistencia.

En este sentido, el trabajo llevado a cabo por proyectos tales como "U-MATIC" en Chile; el rescate del Archivo del CEMA; o la exposición "Vídeo_MAC", que destaca la tarea pionera del Sector de Video; se constituyen como proyectos de investigación multidisciplinar que van más allá de la remediación digital, abriendo la posibilidad de generar nuevas lecturas sobre acervos que de otra manera se desvanecerían de la memoria de las historias del arte regional. Como se ha visto a lo largo de este recorrido, sus esfuerzos favorecen el estudio y difusión de las obras videoartísticas, a través de la conservación y remediación de piezas históricas, la puesta en circulación de estas investigaciones y la ampliación y reformulación de las genealogías del videoarte en el Cono Sur.

\section{Conclusiones}

A lo largo de este escrito se ha procurado reflexionar en las relaciones inestables y complejas establecidas entre el videoarte y los archivos - analógicos y virtualesvinculados a eventos pioneros de videoarte en el Cono Sur. Los casos presentados han procurado hacer tangibles estas problemáticas y visibilizar la necesidad de aspirar a una praxis de archivos que permita proteger este patrimonio audiovisual. Los proyectos de investigación mencionados, por otra parte, evidencian la importancia de contar con fondos y archivos que hagan posible la supervivencia de obras y documentación en torno a la producción videoartística.

En referencia al advenimiento de la cultura en red y el giro digital, se han enfatizado los aspectos positivos del surgimiento de proyectos de investigación y remediación audiovisual, que han permitido articular nuevas narrativas sobre el videoarte de la región. No obstante, las plataformas digitales - surgidas a partir de algunos de estos proyectos - también son efímeras, tal como señala Mariela Cantú internet es "una acumulación de ruinas; de links que no funcionan, de información replicada, de datos desactualizados" (2015, p.99).

Apoyar y estimular el desarrollo de investigaciones en este campo es necesario y complementa - pero no sustituye- la responsabilidad que las instituciones han contraído al albergar fondos documentales y obras audiovisuales. Las instituciones que resguardan estos fondos deberían impulsar acciones para la salvaguarda de ese valioso material patrimonial, articulando proyectos de rescate e investigación conjuntos, que 
permitan la sinergia de fuerzas - y financiamiento- entre instituciones culturales y educativas. Ello permitiría, a la postre, asegurar la continuidad del pasado analógico y digital. En este sentido, se han mencionado aquí, ejemplos elocuentes de que esto es posible.

Por ello sostenemos que el videoarte del sur se encuentra en una encrucijada, un momento en el cual aún es posible acceder a una parte del pasado videográfico analógico. Este pasado resiste en acervos institucionales descuidados y en otros que aún se encuentran activos y parcialmente accesibles. Por este motivo, se hace necesario tomar recaudos para evitar la desaparición definitiva de piezas y documentos que dan bases a genealogías históricas del sur. Gracias a la acción de proyectos con base académico-institucional, ha sido factible recuperar obras patrimoniales $y$ reconstruir historias de gran valía, que se constituyen en ejemplo excepcional de lo que podría, y debería, hacerse. El valor de este tipo de acciones, como muchos investigadores de los new media insisten en afirmar, consiste en que reinscriben el pasado analógico en las coordenadas del presente, y reafirman el compromiso institucional por conservar las obras y asegurar su longevidad.

\section{Referencias}

Alonso, R. (2015). Hacia una genealogía del videoarte argentino. En R. Alonso, Elogio de la Lowtech. Historia y estética de las artes electrónicas en América Latina. Luna Editores, 80-99.

ASSOCIAÇÃO CULTURAL VIDEOBRASIL. (s.f.). Acervo. http://site.videobrasil.org.br/acervo [Consulta: 1 abril 2021]

BOLTER, J.D. y GRUSIN, R. (2000). Remediation. Understanding New Media. MIT Press.

Cantú, M. (2015). Archivos y video: no lo hemos comprendido todo. Cuadernos Del Centro De Estudios De Diseño Y Comunicación, (52), 95-106. https://doi.org/10.18682/cdc.vi52.1332.

CANTÚ, M. (2011). Proyecto ARCA video argentino: base de datos y archivo online de video arte argentino. Arkadin. Estudios sobre cine y artes visuales, 89-91.

CANTÚ, M. et AL. (s.f.). ARCA Video Argentino. http://arcavideoargentino.com.ar/ [Consulta: 18 abril 2021]

CRescentino, A. (2021). El ICl de Buenos Aires. Epicentro del video de creación y vínculos con Uruguay. Revista [sic], https://www.aplu.org.uy/revista-sic

Gallet, P. (2015) Donación de los archivos del Festival Franco Chileno de Video Arte. En Catálogo 12 Bienal de Artes Mediales - Hablar en lenguas, BAM, Santiago de Chile, pp. 22-23.

LA Ferla, J. (2010a). Por una praxis de archivo para las artes tecnológicas experimentales en América Latina. Significação, 37 (33), 43-61, https://doi.org/10.11606/issn.23167114.sig.2010.68103 
LA FerLA, J. (2010b). Memorias audiovisuales posanalógicas y predigitales. Por una praxis de archivos en América Latina. Secuencias, 37 (33), 59-74.

LiÑero AREND, G. (2010) Apuntes para una Historia del Video en Chile. Ocho libros.

MANovich, L. (2001). El lenguaje de los nuevos medios de comunicación. La imagen en la era digital. Paidós Comunicación 163.

MUSEU DE ARTE CONTEMPORÂNEA DA UNIVERSIDADE DE SÃO PAULO (s.f.). Vídeo_MAC http://video.mac.usp.br/en/ [Consulta: 17 abril 2021]

Pato, A. (2014). Arquivos digitais: a experiência do acervo Videobrasil. En G. Beiguelman y A. Gonçalves Magalhães, Futuros possíveis: arte, museus e arquivos digitais. Editora Peirópolis Itda., 86-96.

SARLO, B. (1994). Escenas de la vida posmoderna. Intelectuales, arte y videocultura en la Argentina. Ariel.

SteyerL, H. (2014). En defensa de la imagen pobre. En H. Steyerl, Los Condenados de la Pantalla. Caja negra, pp.33-48.

TADEO FUICA, B. Y BALÁs, M. (2016). CEMA: archivo, video y restauración democrática. FIC-UdelaR, ICAU.

ZUZULICH, J. (2016). La imagen que desborda: viaje, diario y videoarte. Eduntref. 